\title{
Sub-modeling approach for obtaining structural stress histories during dynamic analysis
}

\author{
T. T. Rantalainen, A. M. Mikkola, and T. J. Björk \\ Lappeenranta University of Technology, Department of mechanical engineering, Lappeenranta, Finland
}

Correspondence to: T. T. Rantalainen (tuomas.rantalainen@lut.fi)

Received: 15 November 2012 - Accepted: 26 January 2013 - Published: 11 February 2013

\begin{abstract}
Modern machine structures are often fabricated by welding. From a fatigue point of view, the structural details and especially, the welded details are the most prone to fatigue damage and failure. Design against fatigue requires information on the fatigue resistance of a structure's critical details and the stress loads that act on each detail. Even though, dynamic simulation of flexible bodies is already current method for analyzing structures, obtaining the stress history of a structural detail during dynamic simulation is a challenging task; especially when the detail has a complex geometry. In particular, analyzing the stress history of every structural detail within a single finite element model can be overwhelming since the amount of nodal degrees of freedom needed in the model may require an impractical amount of computational effort. The purpose of computer simulation is to reduce amount of prototypes and speed up the product development process. Also, to take operator influence into account, real time models, i.e. simplified and computationally efficient models are required. This in turn, requires stress computation to be efficient if it will be performed during dynamic simulation. The research looks back at the theoretical background of multibody simulation and finite element method to find suitable parts to form a new approach for efficient stress calculation. This study proposes that, the problem of stress calculation during dynamic simulation can be greatly simplified by using a combination of Floating Frame of Reference Formulation with modal superposition and a sub-modeling approach. In practice, the proposed approach can be used to efficiently generate the relevant fatigue assessment stress history for a structural detail during or after dynamic simulation. Proposed approach is demonstrated in practice using one numerical example. Even though, examples are simplified the results show that approach is applicable and can be used as proposed.
\end{abstract}

\section{Introduction}

Multibody dynamic simulation represents a remarkable improvement in predicting machine performance compared to previous methods, which are often based on very simplified analytical models in combination with large safety margins for model uncertainties or empirical testing. With the development of more computationally powerful computers over recent decades, dynamic simulation has increasingly become a standard tool for comprehensive machine design. Furthermore, this continuously increasing computational power, combined with the availability of increasingly advanced codes, offers more possibilities for the dynamic analysis of complex structures.
Using dynamic simulation to determine stresses for flexible bodies also provides an opportunity to predict the fatigue life of a structure in practical applications. Currently in engineering applications, the prediction of fatigue life for a structure is thought to be a separate stage of design due its complexity and computational burden. If fatigue life prediction is implemented efficiently in multibody codes, it could be used throughout all stages of design without dedicating discrete designing stages to it. These two scenarios of the use of the fatigue analysis are depicted in Fig. 1.

Instead of being an explicit design step, fatigue analysis can be integrated into the design process (Fig. 1 right). This makes fatigue analysis an integrated part of the design process, and from a design point of view, fatigue analysis is 


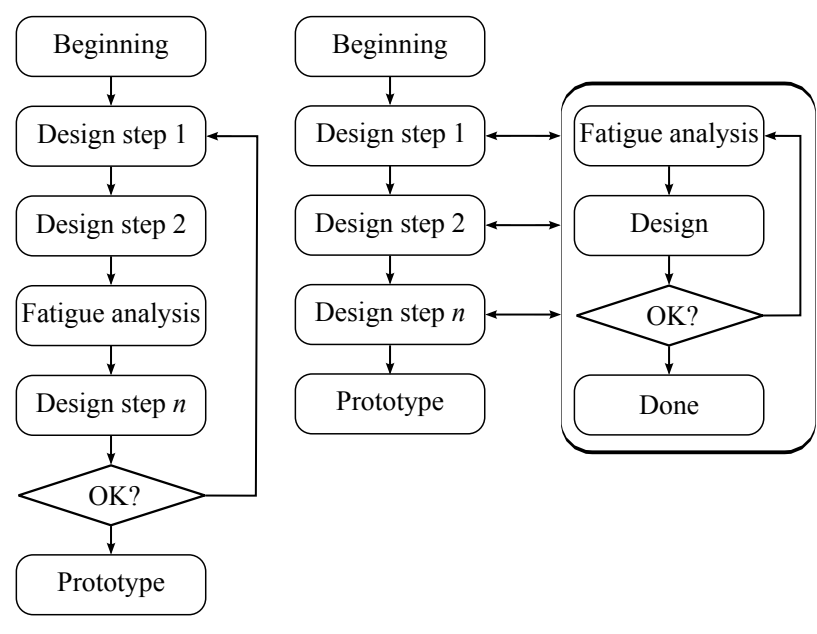

Figure 1. Workflow of a design process with integrated fatigue analysis.

taken into account automatically. To perform mutual integration, efficient methods for performing fatigue analysis are required.

Dynamically loaded structures such as booms are typically manufactured by welding. By definition, fluctuating loads result in fatigue damage to a structure. Without post-weld treatment, welds are prone to fatigue (Maddox, 1991; Haagensen and Maddox, 2011). In dynamic analysis, structural details that do not affect the dynamic behavior of a structure are usually neglected. Typically, this means that stress raisers are not analyzed in dynamic simulation even though they might be a possible location for fatigue. If treated separately, more work is required in fatigue analysis (Fig. 1 left). In Fig. 2, an excavator crane is depicted to illustrate the possible locations that might be vulnerable to fatigue and should be taken into consideration when predicting the fatigue life of a crane.

For stress history prediction, a welded structure requires particular attention. In addition, as computational power increases, there is increasing interest in predicting fatigue life for dynamically loaded structures. Even though computational capabilities have been greatly increased, the need remains for using coordinate reduction methods, especially in the case of large and complicated structures. Multibody dynamic simulation can be used to analyze the dynamics of complex mechanical systems. It can also be used to determine dynamic loads or even stresses for further fatigue analysis. Obtaining stress data for fatigue analysis from multibody system simulation is a main component of this work.

A new strategy developed in the proposed method combines three commonly used engineering approaches: the finite element method, the floating frame of reference formulation, and the sub-modeling approach. This strategy can be used when carrying out dynamic simulation to obtain, efficiently, the stress history of an arbitrary notch. In literature it is shown that computer simulations can be used for fault

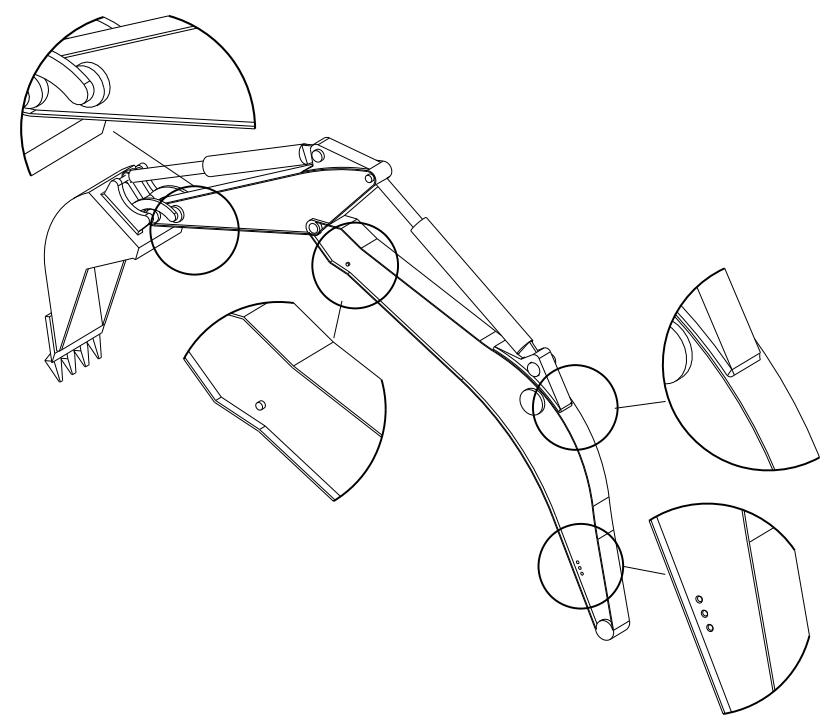

Figure 2. A crane with numerous discontinuities, which can be prone to fatigue.

diagnosis (Korkealaakso et al., 2006). Moreover, this stress history can further be used as initial data for the fatigue analysis. In addition to efficient stress history calculation, the proposed combined methods strategy offers other beneficial features, such as the possibility of attaching structural details to a simulation model without modifying the simulation model. Furthermore, since structural detail does not affect the overall behavior of a simulation, the number of details can be changed arbitrary and separately.

To approximate, efficiently, the fatigue life of a structure or structural detail, some simplifying assumptions must be made. These assumptions decrease the quality of fatigue life estimation, especially in high frequency loading. For this work, a linear strain-stress relationship is assumed. In addition, presumptions have been made related to cumulative damage counting. For example, since fatigue damage can be linearly accumulated, structural failure is predicted when the entire available fatigue life is consumed. The focus here is on linear deformation. The strategy considers plastic deformation only local to the tip of a crack. Material nonlinearities fall outside the scope of this work. However, fatigue life estimation gives useful information when comparing alternative structures.

\section{The floating frame of reference formulation}

The floating frame of reference formulation is typically applicable to systems with large displacements and rotations and small deformations, even though the method can be used for large deformation problems (Wallrapp and Wiedemann, 2003). The method is based on describing the deformations of a flexible body with respect to a frame of reference. With 


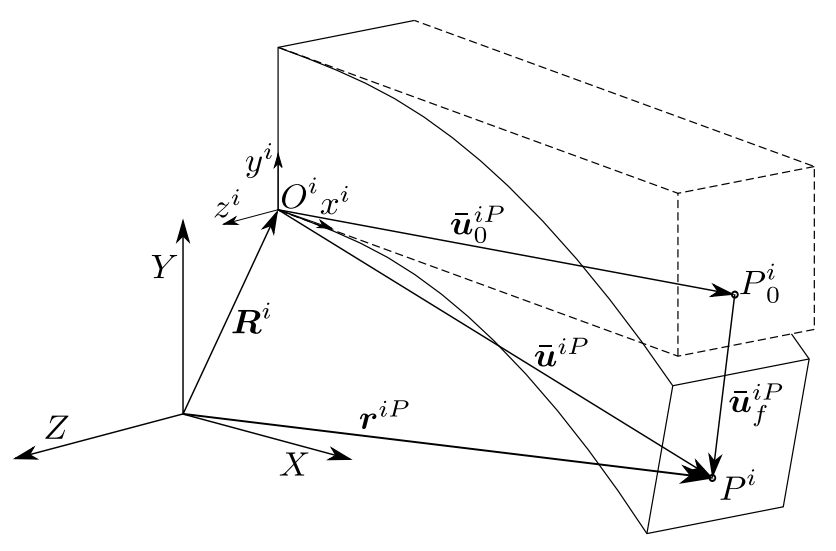

Figure 3. Position of particle $P^{i}$ in a flexible body.

the frame of reference, large displacements and rotations can be described. The deformations of a flexible body in relation to its frame of reference can be described with a number of methods, but in the present study, deformation can be depicted with orthonormalized Craig-Bampton modes (Craig Jr. and Bampton, 1968). In it, eigenmodes are used together with static modes to describe structural deformation. The modes can be obtained using a finite element method.

The formulation separates the deformation of the body from the reference motion. The dynamics of the body can be generated using reference motion that is superposed by the deformation of the body. The interaction between the reference motion and deformation is accounted for with a mass matrix and quadratic velocity vector. This permits even mass distribution and inertia modeling (Shabana, 1998).

Figure 3 illustrates the position of particle $P^{i}$ within a flexible body $i$. In the undeformed state, the position of the particle in the local reference frame of the body can be determined by vector $\overline{\boldsymbol{u}}_{0}^{i P}$.

As body $i$ is deformed (Fig. 3), the position of particle $P^{i}$ changes according to the vector $\overline{\boldsymbol{u}}_{f}^{i P}$. The global reference frame is represented (Fig. 3) using Cartesian coordinates $X$, $\mathrm{Y}$, and Z. Respectively, the local reference frame of body $i$ consists of coordinates $\mathrm{x}^{i}, \mathrm{y}^{i}$, and $\mathrm{z}^{i}$. Therefore, the location of the particle in a global reference frame can be defined with the vector $\boldsymbol{r}^{i P}$ as follows:

$\mathbf{r}^{i P}=\boldsymbol{R}^{i}+\mathbf{A}^{i}\left(\overline{\boldsymbol{u}}_{0}^{i P}+\overline{\boldsymbol{u}}_{f}^{i P}\right)$,

where $\boldsymbol{R}^{i}$ is translation of the local reference coordinate system of body $i$ in the global coordinate system, and matrix $\mathbf{A}^{i}$ is the rotation matrix, which is expressed here in terms of four Euler parameters.

In Eq. (1), $\overline{\boldsymbol{u}}_{0}^{i P}$ is the position vector of particle $P^{i}$ in the local reference coordinate system for the undeformed configuration, and $\overline{\boldsymbol{u}}_{f}^{i P}$ is the position vector in the local reference coordinate system for the deformed configuration. The behavior of the vector $\overline{\boldsymbol{u}}_{f}^{i P}$ can be described with a series of parallel differential equations. By separating the variables, if possible, the equation results in an infinite series that describes the deformations. For computational reasons, the infinite series cannot be applied to the analysis of flexible bodies. In practical application, the vector $\overline{\boldsymbol{u}}_{f}^{i P}$ is described using the finite element method.

The rotation matrix $\mathbf{A}^{i}$ using Euler parameters can be formulated as follows.

$$
\begin{aligned}
& \mathbf{A}^{i}= \\
& {\left[\begin{array}{ccc}
\frac{1}{2}-\left(\theta_{2}^{i}\right)^{2}-\left(\theta_{3}^{i}\right)^{2} & \theta_{1}^{i} \theta_{2}^{i}-\theta_{0}^{i} \theta_{3}^{i} & \theta_{1}^{i} \theta_{3}^{i}+\theta_{0}^{i} \theta_{2}^{i} \\
\theta_{1}^{i} \theta_{2}^{i}+\theta_{0}^{i} \theta_{3}^{i} & \frac{1}{2}-\left(\theta_{1}^{i}\right)^{2}-\left(\theta_{3}^{i}\right)^{2} & \theta_{2}^{i} \theta_{3}^{i}-\theta_{0}^{i} \theta_{1}^{i} \\
\theta_{1}^{i} \theta_{3}^{i}-\theta_{0}^{i} \theta_{2}^{i} & \theta_{2}^{i} \theta_{3}^{i}+\theta_{0}^{i} \theta_{1}^{i} & \frac{1}{2}-\left(\theta_{1}^{i}\right)^{2}-\left(\theta_{2}^{i}\right)^{2},
\end{array}\right]}
\end{aligned}
$$

where $\theta_{0}^{i}, \theta_{1}^{i}, \theta_{2}^{i}$, and $\theta_{3}^{i}$ are Euler parameters. In this study, Euler parameters are used to avoid singular conditions, which can occur when Euler or Bryant angles are used (Nikravesh and Chung, 1982). The following mathematical constraint must be taken into consideration when Euler parameters are applied.

$\left(\theta_{0}^{i}\right)^{2}+\left(\theta_{1}^{i}\right)^{2}+\left(\theta_{2}^{i}\right)^{2}+\left(\theta_{3}^{i}\right)^{2}=1$

The first time-derivative of the Euler parameters $\dot{\theta}$ and the angular velocity vector $\bar{\omega}^{i}$ has the following linear connection.

$\overline{\boldsymbol{\omega}}^{i}=\overline{\mathbf{G}}^{i^{\mathrm{T}}} \dot{\boldsymbol{\theta}}^{i}$

Matrices $\mathbf{A}^{i}$, and $\overline{\mathbf{G}}^{i}$ depend on the selected generalized coordinates. Using Euler parameters, the matrix $\overline{\mathbf{G}}^{i}$ can be expressed as.

$\overline{\mathbf{G}}^{i}=\left[\begin{array}{cccc}-\theta_{1}^{i} & \theta_{0}^{i} & \theta_{3}^{i} & -\theta_{2}^{i} \\ -\theta_{2}^{i} & -\theta_{3}^{i} & \theta_{0}^{i} & \theta_{1}^{i} \\ -\theta_{3}^{i} & \theta_{2}^{i} & -\theta_{1}^{i} & \theta_{0}^{i}\end{array}\right]$,

where $\overline{\mathbf{G}}^{i}$ is the transformation matrix that relates the angular velocity $\bar{\omega}^{i}$ of a body and the first time derivative of the Euler parameter. Using the model reduction method, the position of an arbitrary particle $P^{i}$ in the global coordinate system can be expressed as.

$\boldsymbol{r}^{i P}=\boldsymbol{R}^{i}+\mathbf{A}^{i}\left(\overline{\boldsymbol{u}}_{0}^{i P}+\boldsymbol{\Phi}_{R}^{i P} \boldsymbol{p}^{i}\right)$

Equation (6) is determined using a collection of modes. The vector $\overline{\boldsymbol{u}}_{0}^{i P}$ and the modal matrix $\boldsymbol{\Phi}_{R}^{i P}$ are constant with time. Consequently, they only need to be calculated once, at the beginning of the simulation.

The finite element model often consists of a large number of nodal degrees of freedom, and the use of large finite element models to describe flexibility may be computationally inefficient. For this reason, the floating frame of reference formulation is often used together with a modal reduction method in which the deformation is described with structural modes. Modes may be the presumed forms of deformation, 
but most often, they are eigenmodes of structural vibrations. The eigenmodes can be obtained from a finite element model of the structure. By employing a modal reduction method, the deformation vector $\overline{\boldsymbol{u}}_{f}^{i P}$ can be expressed in modal coordinates with a shape matrix.

$\overline{\boldsymbol{u}}_{f}^{i P}=\boldsymbol{\Phi}_{R}^{i P} \boldsymbol{p}^{i}$

$\boldsymbol{\Phi}_{R}^{i P}$ is the modal matrix whose columns describe the translation of particle $P^{i}$ within the assumed deformation modes of the flexible body $i$ (Shabana, 2005), and $\boldsymbol{p}^{i}$ is a vector of elastic coordinates. In general, the complete modal matrix $\boldsymbol{\Phi}^{i P}$ for body $i$ obtained from the finite element method contains the location translation and orientation of particle $P^{i}$. In multibody dynamics, the modal matrix should separate translation and orientation descriptions into their own components.

The orthogonal shape matrix can be formulated from the eigenmodes of the body. Typically, the shape superposition technique yields acceptably accurate results even though only a few differential equations are applied. By approximating Eq. (7) with an $n_{p}$ number of modal coordinates, the deformation vector $\overline{\boldsymbol{u}}_{f}^{i P}$ for a particle $P^{i}$ can be written as follows.

$\overline{\boldsymbol{u}}_{f}^{i P} \approx \sum_{j=1}^{n_{p}} \boldsymbol{\varphi}_{R, j}^{i} p_{j}^{i}=\boldsymbol{\Phi}_{R}^{i P} \boldsymbol{p}^{i}$

$p_{j}^{i}$ is one modal coordinate in the modal coordinate vector that corresponds to the modal shape $j$. Rotations due to body deformation do not have any direct use in the floating frame of reference formulation, and therefore they are usually ignored. However, rotation modes can be used in the description of constraint equations applied to rotational degrees of freedom (Korkealaakso et al., 2009). Rotational modes are used here to connect sub-models to large-scale models. With the rotational modal matrix $\boldsymbol{\Phi}_{\theta}^{i P}$, the rotation change $\varepsilon_{f}^{i P}$ resulting from deformation can be approximated as follows.

$\boldsymbol{\varepsilon}_{f}^{i P} \approx \sum_{j=1}^{n_{p}} \boldsymbol{\varphi}_{\theta, j}^{i} p_{j}^{i}$

A rotation matrix $\mathbf{A}_{f}^{i P}$ that describes orientation due to deformation at the location of particle $P^{i}$ with respect to the reference frame can be composed like this.

$\mathbf{A}_{f}^{i P}=\mathbf{I}+\tilde{\boldsymbol{\varepsilon}}_{f}^{i P}$

I is $(3 \times 3)$ identity matrix. The $~$ symbol above a variable indicates the skew-symmetric form. The orientation at the location of particle $P^{i}$ within the frame of reference can be expressed as follows.

$\overline{\boldsymbol{v}}_{f}^{i P}=\mathbf{A}_{f}^{i P} \overline{\boldsymbol{v}}_{0}^{i P}$

$\overline{\boldsymbol{v}}_{0}^{i P}$ is the orientation of the location of particle $P^{i}$ in the undeformed state. The description of the rotation of the node has no direct use in formulating equations of motion, but the rotation may be needed to describe the constraint equations that are applied to rotational degrees of freedom. Taking into account the relation between first time derivative of Euler angels and angular velocity of the body $i$, Eq. (4), the generalized velocity vector of the flexible body $i$ can be written as follows.

$\dot{\boldsymbol{q}}^{i}=\left[\begin{array}{lll}\dot{\boldsymbol{R}}^{i^{\mathrm{T}}} & \overline{\boldsymbol{\omega}}^{i^{\mathrm{T}}} & \dot{\boldsymbol{p}}^{i^{\mathrm{T}}}\end{array}\right]$

The velocity of particle $P^{i}$ can be determined by differentiating Eq. (1) with respect to time as follows.

$\dot{\boldsymbol{r}}^{i P}=\left[\begin{array}{ccc}\mathbf{I} & -\mathbf{A}^{i} \overline{\mathbf{G}}^{i} \tilde{\bar{u}}_{f}^{i P} & \mathbf{A}^{i} \boldsymbol{\Phi}_{R}^{i P}\end{array}\right]\left[\begin{array}{c}\dot{\boldsymbol{R}}^{i} \\ \dot{\boldsymbol{\theta}}^{i} \\ \dot{\boldsymbol{p}}^{i}\end{array}\right]$

Note the vector, in the right hand of Eq. (13), describes the velocity of the generalized coordinates of a flexible body $i$. Differentiating the velocity of a particle Eq. (13) with respect to time, the acceleration of a particle can be written in this manner.

$$
\begin{aligned}
\ddot{\boldsymbol{r}}^{i P} & =\left[\begin{array}{lll}
\mathbf{I} & -\mathbf{A}^{i} \tilde{\overline{\boldsymbol{u}}}^{i P} \overline{\mathbf{G}}^{i} \mathbf{A}^{i} & \boldsymbol{\Phi}_{R}^{i P} \boldsymbol{R}^{i P}
\end{array}\right]\left[\begin{array}{c}
\ddot{\boldsymbol{R}}^{i} \\
\ddot{\boldsymbol{\theta}}^{i} \\
\ddot{\boldsymbol{p}}^{i}
\end{array}\right] \\
& +\left[\begin{array}{lll}
\mathbf{0} & -\mathbf{A}^{i} \tilde{\overline{\boldsymbol{\omega}}}^{i} \tilde{\overline{\boldsymbol{u}}}^{i P} \overline{\mathbf{G}}^{i} & 2 \mathbf{A}^{i} \tilde{\overline{\boldsymbol{\omega}}}^{i} \boldsymbol{\Phi}_{R}^{i P}
\end{array}\right]\left[\begin{array}{c}
\dot{\boldsymbol{R}}^{i} \\
\dot{\boldsymbol{\theta}}^{i} \\
\dot{\boldsymbol{p}}^{i}
\end{array}\right],
\end{aligned}
$$

where $\ddot{\boldsymbol{R}}^{i}, \ddot{\boldsymbol{\theta}}^{i E}$, and $\ddot{\boldsymbol{p}}^{i}$ are accelerations of translational coordinates, Euler parameters, and modal coordinates of body $i$.

According to the D'Alembert principle, inertial forces can be treated as external forces, thus forces of the body $i$ can be written as follows.

$\boldsymbol{F}^{i}=\int_{V^{i}} \rho^{i} \ddot{\boldsymbol{r}}^{i P} \mathrm{~d} V^{i}$,

where $\rho^{i}$ is density and $V^{i}$ is the volume of a body $i$, respectively. The virtual work done by the inertial forces can be represented as.

$\delta W^{i}=\int_{V^{i}} \rho^{i} \delta \boldsymbol{r}^{i P \mathrm{~T}} \ddot{\boldsymbol{r}}^{i P} \mathrm{~d} V^{i}$

The virtual displacement of the position vector $\delta r^{i P}$ can be expressed as.

$\delta \boldsymbol{r}^{i P}=\frac{\delta \boldsymbol{r}^{i P}}{\delta \boldsymbol{q}^{i}} \delta \boldsymbol{q}^{i}=\left[\begin{array}{lll}\mathrm{I} & -\mathbf{A}^{i} \tilde{\overline{\boldsymbol{u}}}^{i P} \overline{\mathbf{G}}^{i} \mathbf{A}^{i} & \boldsymbol{\Phi}_{R}^{i P}\end{array}\right] \delta \boldsymbol{q}^{i}$,

By substituting the virtual displacement Eq. (17) into the equation for virtual work (Eq. 16) and separating terms, the following equation can be obtained.

$\delta W^{i}=\delta \boldsymbol{q}^{i}\left[\mathbf{M}^{i} \ddot{\boldsymbol{q}}^{i}+\boldsymbol{Q}^{i v}\right]$, 
where $\delta \boldsymbol{q}^{i}$ is virtual change of the generalized coordinates, $\boldsymbol{Q}^{i v}$ is quadratic velocity vector of body $i$, and $\mathbf{M}^{i}$ is the mass matrix.

The virtual work of externally applied forces can be defined as.

$\delta W^{i e}=\int_{V^{i}} \delta \boldsymbol{r}^{i P^{\mathrm{T}}} \boldsymbol{F}^{i P} \mathrm{~d} V^{i}=\delta \boldsymbol{q}^{i \mathrm{~T}} \boldsymbol{Q}^{i e}$,

where $\boldsymbol{F}^{i P}$ is externally applied force per unit volume, and $\boldsymbol{Q}^{i e}$ is the vector of generalized forces, which can be expressed as follows.

$\boldsymbol{Q}^{i e}=\left[\begin{array}{c}\boldsymbol{Q}_{R}^{i e} \\ \boldsymbol{Q}_{\theta}^{i e} \\ \boldsymbol{Q}_{p}^{i e}\end{array}\right]$,

where $\boldsymbol{Q}_{R}^{i e}$ is translational components, $\boldsymbol{Q}_{\theta}^{i e}$ is rotational components, and $\boldsymbol{Q}_{p}^{i e}$ is elastic components of the generalized force vector, respectively.

The elastic forces can be described using modal coordinates and the stiffness matrix in modal coordinates $\mathbf{K}^{i}$. The stiffness matrix in modal coordinates can be obtained using component mode synthesis. The virtual work of the elastic forces can be expressed as.

$\delta W^{i f}=\delta \boldsymbol{p}^{\mathrm{T}} \mathbf{K}^{i} \boldsymbol{p}^{i}$

The vector of elastic forces can be represented as follows.

$\boldsymbol{Q}^{i f}=\left[\begin{array}{c}\mathbf{0} \\ \mathbf{0} \\ \mathbf{K}^{i} \boldsymbol{p}^{i}\end{array}\right]$

In multibody dynamics, different types of joints between bodies are accounted for with kinematic constraints applied on generalized coordinates. Algebraic equations are used for the description of constraints between bodies. By examining only holonomic constraints, constraint equations can be expressed as follows.

$C(q)=0$

where, $\boldsymbol{C}$ is the constraint vector for the system. Equations of motion may be formulated using the widely known Lagrange method, in which kinematic constraints are accounted for as supplementary algebraic equations with the help of Lagrange multipliers. The method is called global formulation since it does not differentiate between open and closed kinematic chains, as topological methods do. After employing the concept of virtual work to externally applied forces and then introducing constraints with help of Lagrange multipliers, the equation of motion can be written in the form of a differential algebraic equation (DAE).

$\mathbf{M} \ddot{\boldsymbol{q}}+\mathbf{K} \boldsymbol{q}+\mathbf{C}_{\boldsymbol{q}}^{\mathrm{T}} \boldsymbol{\lambda}=\boldsymbol{Q}^{e}+\boldsymbol{Q}^{v}-\boldsymbol{Q}^{f}$
Equations (23) and (24) form a set of differential algebraic equations, which can be converted to ordinary differential equations (ODE) to solve for the dynamic response of the multibody system in the time domain. To be able to apply traditional ODE solvers to the system of equations, the constraint equations must be differentiated twice with respect to time.

$\ddot{\boldsymbol{C}}(\boldsymbol{q}, \dot{\boldsymbol{q}}, \ddot{\boldsymbol{q}})=\mathbf{C}_{q} \ddot{\boldsymbol{q}}+\left(\mathbf{C}_{q} \dot{\boldsymbol{q}}\right)_{\boldsymbol{q}} \dot{\boldsymbol{q}}=\mathbf{0}$,

where $\boldsymbol{Q}^{c}=-\left(\mathbf{C}_{q} \dot{\boldsymbol{q}}\right)_{q} \dot{\boldsymbol{q}}$ is the constraint force vector for the system. As a result, the final matrix form of equations of motion describing the system dynamics looks as following.

$\left[\begin{array}{cc}\mathbf{M} & \mathbf{C}_{\boldsymbol{q}}^{\mathrm{T}} \\ \mathbf{C}_{\boldsymbol{q}} & \mathbf{0}\end{array}\right]\left[\begin{array}{c}\ddot{\boldsymbol{q}} \\ \lambda\end{array}\right]=\left[\begin{array}{c}\boldsymbol{Q}^{e}+\boldsymbol{Q}^{v}-\boldsymbol{Q}^{f} \\ \boldsymbol{Q}^{c}\end{array}\right]$

\subsection{Fatigue}

Fatigue is a failure that occurs after cyclic loading, and it is a common cause of structural fracture. Fatigue damage is one of the most common faults in dynamically loaded structures. In principle, the entire development of fatigue damage can be described as follows: one or more cracks form in the material, and the cracks grow until fatigue failure takes place.

A fundamental design objective for any dynamically loaded structure exposed to cyclic loading or vibration is to avoid fatigue failure throughout its service life. Welding is one of the most efficient methods used to manufacture structures. Cranes, vehicle frames, and machines are just some examples of welded structures that are dynamically loaded. As a structural detail, a weld is initially very prone to fatigue due to the notch effect, high tensile residual stresses, and welding flaws. High-strength steels, which are seeing increasing use, are even more sensitive to this phenomenon. In general, high strength steels are chosen to achieve larger payloads with more slender structural elements. As a trade off, the slender structures are subject to increased nominal stresses and welds become more prone to fatigue due to the higher stresses. Typically, structural welds are at or near areas of structural discontinuity. The weld itself is a local discontinuity. Furthermore, welding processes typically introduce flaws in the weld or weld toe such as undercuts, the inclusion of impurities, and cold laps. These flaws are sources of incipient cracking.

Empirical testing with actual parts or complete systems is time consuming and laborious. Traditionally, the approach to avoiding fatigue failures in a new system is to fatigue test specific structural details before integrating them into the system design. In fact, many kinds of typical welded details can be found commonly in the literature including fillet welds, corner joints, and butt joints. This approach has several weaknesses. For example, all pertinent structural details to be used should be tested under various loading conditions if the fatigue evaluation is to be comprehensive, and the 
approach ignores other parameters that may relate to a particular joint, such as the number of weld beads or other geometrical and technical details. Obviously, for a complex system with an arbitrary number of structural details subjected to various multi-axial loading scenarios, it is practically impossible to use this traditional approach.

More recently, numerical methods have been developed to estimate fatigue life making empirical testing unnecessary and allowing the designer to more effectively consider the effects of fatigue (Haagensen and Maddox, 2011). Today, the finite element method has become a standard approach for estimating the fatigue life of a structure. Nonetheless, even though computational capacity is increasing all the time, applying the finite element approach to a complex structure subject to dynamic multi-axial loading presents an overwhelming computational burden.

Fatigue design approaches can be differentiated according to how cracks initiate. In some applications, such as rotating axles, fatigue life is equivalent to the duration of the crack initialization stage. Because of the notch effect, the crack in these applications quickly results in failure. For larger structures, such as machine frames or many welded structures, cracks are present from the beginning, so fatigue life is the determined by the length of time it takes the initial cracks to propagate.

The fatigue life of a structure under dynamic load can be estimated by assuming it to have some initial amount of fatigue endurance and then assuming that one load cycle will result in fatigue damage of some amount. This is commonly known as Palmgren-Miner's rule (Miner, 1945). It was suggested that fatigue damage could be accumulated linearly for a certain amplitude value. Finally, when all fatigue endurance has been depleted, failure is expected. A large amount of fatigue test data can be found in the literature, and stress histories can be obtained through experimentation of by simulation.

\section{Sub-modeling}

A common cause of structural damage is local stress concentration due to structural geometry. Practically all structural damage occurs where one or more stress raisers are present. Problematic details are often combinations of several geometries that concentrate hazardous stresses. Stress raisers result from local discontinuities in real structural features; such as welds, attachments grooves or holes. Typically, small discontinuities are neglected in multibody simulation, since their effect on overall behavior is relatively small compared to their contribution to computational burden. Traditionally, the problem of stress raisers is solved by calculating nominal stress levels and then taking into account the effect of stress raisers by applying predefined stress concentration factors. The stress concentration factor concept cannot be a general approach, since it is obvious that all possible geomet-

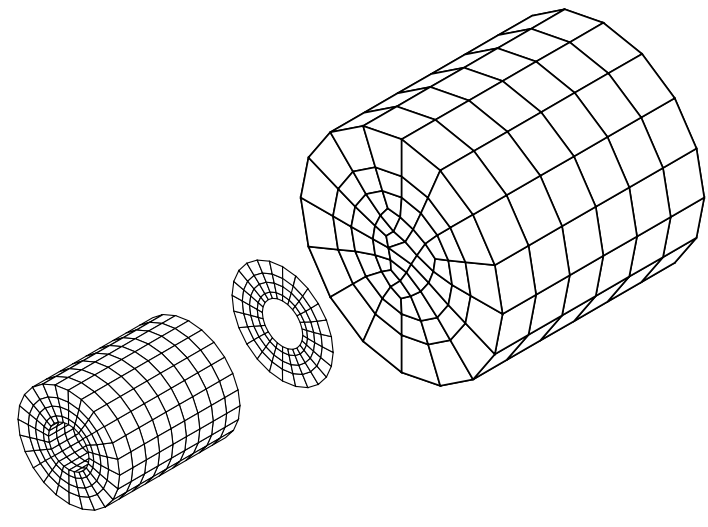

Figure 4. The sub-modeling approach for attaching dissimilar meshes.

rical shapes and their combinations combined with complex loading cases cannot be predefined, especially if a specific but arbitrary level of accuracy is needed. Obviously, the general approach covering all kinds of geometrical combinations in the finite element method is to model them as they appear in the structure. Since stress values change drastically in the neighborhood of a structural discontinuity, a refined element mesh is required, which will lead to a large number of degrees of freedom. This approach is impractical due to the computational burden, especially in the case of multibody simulation.

The sub-modeling approach is commonly used, and it can overcome the previously mentioned problems. With the approach some new problems arise, but they will be discussed later. In principal, a sub-model is a model inside of or on top of a large scale model that describes a certain portion of the large scale model. It can be used to attach a locally refined element mesh to the larger scale model, which does not need to be changed. In sub-modeling, the simplified structural model is complemented by a more refined sub-model of structural details. The sub-models do not influence the operation of the system but get their boundary conditions and loading data from the larger simplified model. The sub-modeling approach is also referred to in the literature as the global/local approach (Knight et al., 1991). The sub-modeling approach can be used to connect dissimilar meshes together as shown schematically in Fig. 4.

Sub-modeling is an approach that is used together with the finite element method to combine two different finite element meshes. There are several reasons the sub-modeling approach is powerful. It can be used to connect finite models into a larger assembly. The approach does not require meshes to be similar and even element types can differ. Currently available methods do not require any coincident nodes. These beneficial features can be utilized to combine separately constructed models or refine the element mesh in a certain area without taking care of refining the mesh smoothly. In addition, a sub-model can be changed easily without modifying 
other parts of the model. Problems arise if the level of refinement differs significantly between two different models. A coarse mesh tends to be too stiff and displacements are underestimated, and if those underestimated displacements are used as boundary conditions for the refined model, calculated stress levels will be non-conservative.

Coupling between the sub-model and the large-scale model is assumed to be one directional, i.e., it is assumed that the behavior of the reduced model in dynamic simulation is not affected by the sub-model. That means, the large scale model is complemented with a sub-model of the desired detail and it does not affect the system's overall stiffness. This crucial simplification makes dynamic simulation and stress calculation independent from each other. Therefore, the computation can be straight forwardly parallelized. Displacement boundary conditions of the sub-model, however, are acquired from the large-scale model. In the proposed approach, during dynamic analysis the general behavior of the structure is calculated with a simplified model and details are examined as a separate problem. For assessing fatigue loads on a structure, this assumption is sufficient since any significant change in structural flexibility due to crack growth occurs only very late in the total life of a structure.

A sub-model can make the overall structure stiffer, or it can only carry boundary conditions without affecting the stiffness of a large-scale structure. Sub-models describe structural details, and only those that are interesting from a design point of view can be attached to a flexible multibody model. Connections between a multibody model and its sub-models are one-directional, guaranteeing that the multibody simulation is not affected by the sub-model. For instance, if a flexible multibody model is a part of a real-time simulation, it will be a real-time simulation even if sub-models are active. In this paper, one way of attaching sub-models to the larger multibody model is introduced, but the concept is general.

\section{Stress in multibody dynamic simulation}

Dimensioning components require information about loading and more accurately, stresses. Even though, stresses can somehow be obtained from rigid body dynamics by using simulated forces as force boundary conditions in finite element method. In general, to obtain structural stresses, structural flexibility should be taken into account. Concept of multibody dynamics gives attractive approach to simulate real operating conditions and thus obtain realistic loading conditions.

Stress recovery methods for a flexible multibody system can be divided into two main categories. One is the stressmode-based method, which determines the body's stress state using a linear combination of stress modes and elastic coordinates. The other is the finite-element post-processing method, in which stresses are calculated by a finite-element code using forces or displacements obtained from multibody simulation. Both approaches have benefits, but in general, the method based on finite-element post processing is more accurate (Arczewski and Frączek, 2005).

For lightweight structures loaded by dynamic forces, accurate dynamic simulation is necessary to guarantee longterm reliability (fatigue life), the accuracy of control, and system usability. Deformation, even small deformations, must be taken into account to achieve the needed simulation precision. Arriving at an optimized design and understanding precisely how internal stresses vary over time leads to structures with improved fatigue life and whole systems that are safer. In the Floating Frame of Reference Formulation, bodies are loaded by numerous unique loads and moments; external forces, constraint forces, and inertial forces, for example. Forces produce deformation, and deformations set up internal stresses. The prediction of local stresses using dynamic simulation reveals structural weaknesses in the early design stages. In addition, dynamic simulation can analyze stress peaks in extreme cases, such as random overloading or component failure.

A stress history from a multibody dynamics simulation can be used as initial data for component dimensioning. Furthermore, it can provide loading data for the analyst that is otherwise difficult to obtain. Finally, the stress history can be used as input for the fatigue analysis of the component. In such cases, one should make sure that simulated operations describe the operating conditions of the machine with sufficient accuracy. With simulation, it is difficult to describe the impact of statistical issues, such as component wear and operator usage habits, on component loading. On the other hand, simulation helps to understand the causes and effects related to loading. This allows the use of optimization routines in component dimensioning. Simulation and measurement on a real-life machine can thus be considered to support each other, and using them together can help to reach an optimal solution.

The literature provides a number of alternative approaches to determining stress histories from multibody simulation. The first to combine the multibody dynamic approach and stress calculation was Melzer (1996). Yim and Lee (1996) obtained dynamic stress histories by using constraint forces solved in a multibody system. Dietz et al. (1997) described an approach for using multibody simulation to obtain all forces for finite element analysis. They also selected the most severe time steps for which stresses of the complete structure were later analyzed in finite element code. Later, Dietz et al. (1998) combined multibody simulation and fatigue life prediction. They obtained the load history from multibody simulation and calculated stress histories for selected locations using a stress load matrix. Stress histories were analyzed in a post-processing stage to predict fatigue life. Dietz (1999) presented a systematic way of combining multibody dynamic simulation and fatigue analysis using stress component modes. Claus (2001) generalizes the deformation-based stress recovery approach to multipurpose 
finite element codes. More recently, Jun et al. (2008) used the modal stress recovery approach to obtain stress for fatigue analysis. They also discussed the reliability of fatigue life calculation. Lee et al. (2009) studied the fatigue life for various parts of a guideway vehicle by coupling multibody dynamics and fatigue analysis. They determined stresses using the modal stress method or quasi-static force method depending on the loading conditions of the part. Braccesi and Cianetti (2005) used a modal approach to recover stresses. Arczewski and Frạczek (2005) compared and discussed differences between force-based and deformation-based stress recovery methods in MBS. More recently, Tobias and Eberhard (2011) obtained stresses using a reduced MBS model and stress modes. They concluded the stress state in any particular point of a flexible body could be expressed as a linear combination of global shape functions for stresses and nodal coordinates.In experiments, fatigue life prediction is mainly related to uniaxial cyclic loading. This leads to discussion about damage hypothesis, and the question arises about which damage hypothesis should be used. This work focuses on welded structures, which can be studied under the assumption of cumulative damage counting. Welded structures without any post-weld treatments have large tensile residual stresses, even nominally as large as the yield strength of the material. In this case, initial compressive loading closes an incipient crack and residual stresses open it again. This leads to a situation in which even a fully compressive loading cycle will result in full fatigue damage. For the proposed method, linear hot spot extrapolation (Poutiainen et al., 2004) is selected, for both simplicity and robustness in use.

\section{Empirical example}

Fatigue damage typically originates from the points of discontinuity of the structure, especially if there is residual stress affecting the discontinuity. In order for the machine system to be simulated in real time, it must often be simplified, and details irrelevant in terms of structural stress neglected. The modeling of small details, such as welded clamp for a hydraulic pipe of a boom, increases the need for computational efficiency, and such details have only a localized importance. To illustrate the method for obtaining stress history of a structural detail, a practical example of a simple crane is studied. The crane and the structural detail are depicted in Fig. 5. In this example the simplified dynamic simulation and relative accurate model of notch are combined together using sub-modeling techniques.

The crane in Fig. 5 is hydraulically driven crane with one degree of freedom. Hydraulic system is modeled using the theory of lumped fluid (Watton, 1989), in which hydraulic system is divided into separate volumes in where pressure is equally distributed.

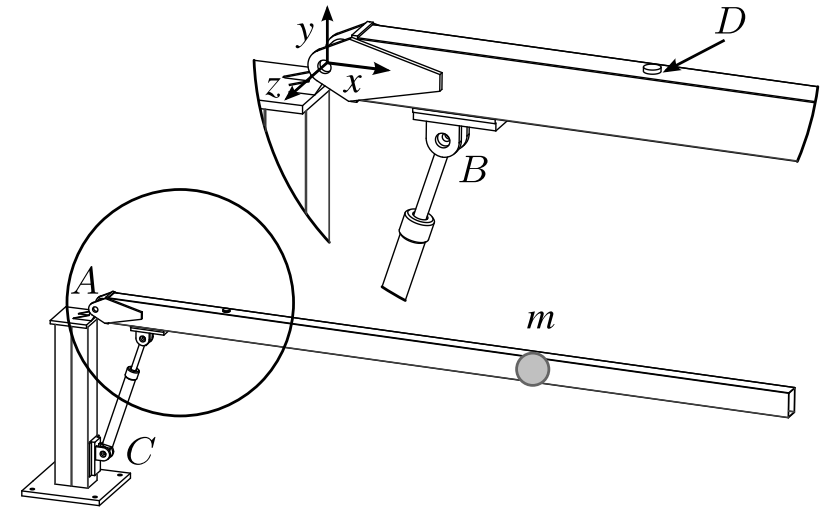

Figure 5. Crane parameters and the placement of strain gauges $(D)$ - Letters $A, B$, and $C$ refer to the joint locations and $m$ is the added mass of $110 \mathrm{~kg}$. Strain gauges are attached close to the welded notch so the first is $0.4 t$ and the second is $1.0 t$ from the notch. Plate thickness $t$ is $4 \mathrm{~mm}$.

A fundamental motivation of the introduced approach is to keep the dynamic simulation as numerically efficient as possible. Therefore, the hosting structural model is simplified and is then reduced with the Craig-Bampton method (Craig Jr. and Bampton, 1968). Even though the proposed approach is general, in this example boom-type structures that can be efficiently described with beam elements are studied. With beam models, obtaining boundary conditions for sub-models is straightforward. In the proposed approach, multibody simulation is used for producing displacement data for the submodel, which is then analyzed and fatigue data is obtained.

Problem about computational burden is tried to overcome by combining sub-modeling and multibody dynamic approach in order to join computationally efficiency of multibody dynamic approach and accuracy of finite element method in observing damaging loads. Sub-model of the crane is shown in Fig. 6. Black circle shows the area where hot spot stress is obtained. Nodes used in hot spot extrapolation are show in Fig. 7.

The beam model represents the center line of a structural component. The sub-model is attached to the interpolated locations of the reduced model via rigid and massless beams. Due to the use of rigid beam webs, the cross-section is assumed to remain planar at the boundary condition points. The effect of this assumption, with respect to stresses in notch, is negligible due to Saint-Venant's principle (von Mises, 1945). In dynamic simulation, translational and rotational displacements are solved as boundary conditions for the sub-model. In general, sub-models are attached at arbitrary locations of the structural component, thus nodal displacement interpolation should be used. 


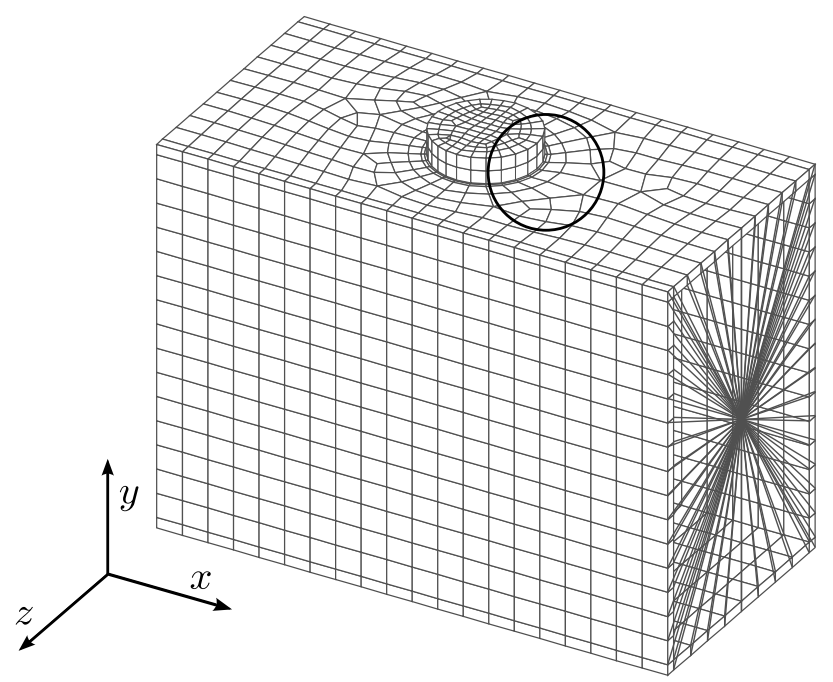

Figure 6. FE-model of the sub-model - the black circle indicates the location of the hot-spot nodes.

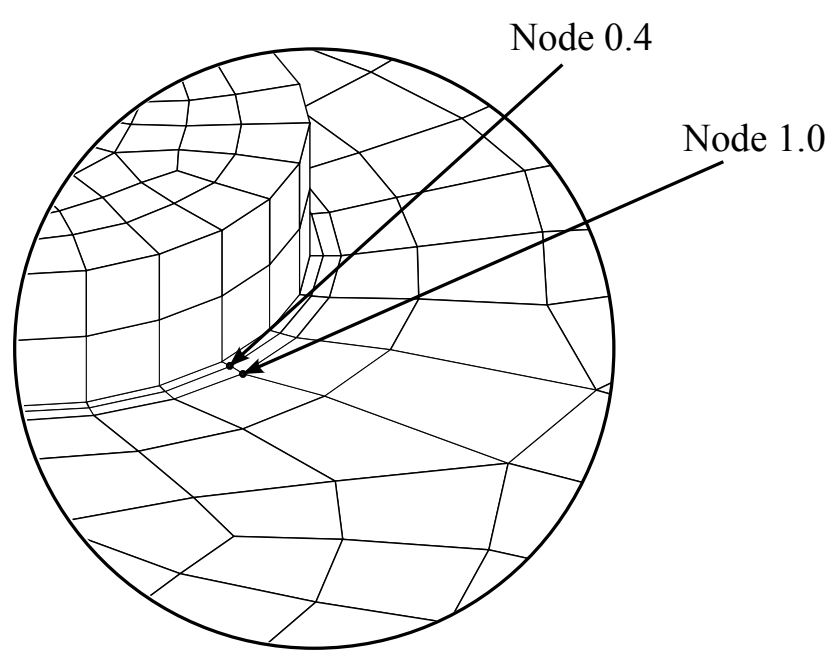

Figure 7. Hot spot extrapolation nodes on a sub-model.

\subsection{Crane model composition and work cycle}

The crane model contains four bodies, which are the crane, support and hydraulic cylinder and shaft for a cylinder. The lenght $L$ of the crane is $4.5 \mathrm{~m}$. depicted in Fig. 5. In addition to those parts model has added mass $m=100 \mathrm{~kg}$ and a hydraulic system. Hydraulic circuit can be neglected while comparing results, since simulation model was driven by predefined movement of the cylinder that is shown in Fig. 8. In Table 1, relevant dimensions and distances from revolute joint $A$ along coordinates $\mathrm{x}, \mathrm{y}, \mathrm{z}$, are presented.

The crane model consists of a beam model with 20 nodes. Craig-Bampton method is used to reduce coordinates of the beam model. Two connection nodes were selected as a masted nodes for Craig-Bampton. Structural flexibility was described using 10 lowest deformation modes. Geometri-
Table 1. Dimensions of the crane.

\begin{tabular}{lll}
\hline Item & x coordinate $[\mathrm{m}]$ & $\mathrm{y}$ coordinate $[\mathrm{m}]$ \\
\hline Revolute joint $A$ & 0 & 0 \\
Revolute joint $B$ & 0.32 & -0.125 \\
Revolute joint $C$ & 0 & -0.925 \\
Mass $m$ & 2.5 & 0 \\
Welded detail $D$ & 0.65 & 0.075 \\
Gauge 0.4 & 0.672 & 0.075 \\
Gauge 1.0 & 0.674 & 0.075 \\
\hline
\end{tabular}

Table 2. Geometrical properties of cross section of the crane and material properties of the crane.

\begin{tabular}{lll}
\hline Property & Value & Unit \\
\hline Profile height & 0.15 & $\mathrm{~m}$ \\
Profile width & 0.10 & $\mathrm{~m}$ \\
Profile area & $1.9 \times 10^{-3}$ & $\mathrm{~m}^{2}$ \\
Plate thickness & 4 & $\mathrm{~mm}$ \\
Area moment of inertia $(y y)$ & $6.17 \times 10^{-6}$ & $\mathrm{~m}^{4}$ \\
Area moment of inertia $(z z)$ & $3.29 \times 10^{-6}$ & $\mathrm{~m}^{4}$ \\
Elastic modulus & 210 & $\mathrm{GPa}$ \\
Poisson's ratio & 0.3 & \\
Density & 7850 & $\mathrm{~kg} \mathrm{~m}^{-3}$ \\
\hline
\end{tabular}

cal properties of cross section of the crane are presented in Table 2.

\subsection{Stress history}

This displacement data is then used as a boundary condition for the sub-model. The sub-model contains the stress concentrations where fatigue damage can possibly occur. Nodal displacement history is applied as boundary conditions on the sub-model as a sequential set of static boundary conditions. The finite element mesh of the sub-model has 1800 linear, brick elements and 250 rigid, massless beams.

The gray lines seen on right side, in Fig. 6, represent rigid and massless beam webs and connect the cross section of the sub-model to the dynamical model via attachment nodes. Two attachment nodes are located on the middle line of the cross section. The use of rigid beam webs keeps the boundary cross section of the sub-model planar. This clearly simplifying assumption is made because in the beam model the cross section is assumed to remain planar. Generally, the problems with beam elements are the higher order deformations of cross section, such as warping and distortion, which are not included in low order beam elements.

Since the sub-model and the dynamic model have overlapping nodes, boundary conditions for the sub-model can be fixed based on nodal deformation from the dynamic simulation. In the case of non-overlapping nodes, interpolation of nodal translational deformation and rotation deformation between nodes is required. In this case, interpolation could be 


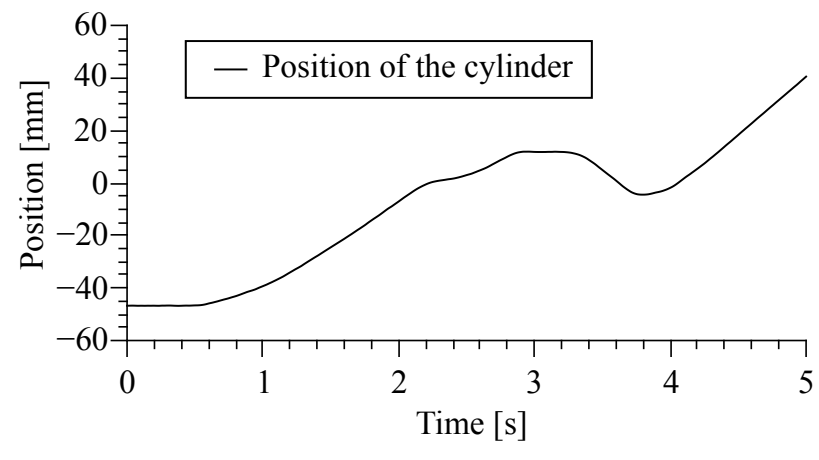

Figure 8. Measured position of the shaft of the hydraulic sylinder.

made linearly between the nodes. Since the sub-model only uses displacements obtained from the dynamic simulation as boundary conditions, it does not interfere with the overall behavior of the model.

For fatigue assessment the hot spot or structural stress is often used (Niemi et al., 2006). The crane was loaded using measured position of a hydraulic cylinder, shown in Fig. 8 A linear surface extrapolation for hot spot stress (Poutiainen et al., 2004) has been performed for obtaining stress histories shown in Fig. 9. The hot spot structural stress at the edge of the welded discontinuity, is based on a linear extrapolation of surface stresses at nodes $4 \mathrm{~mm}$ and $10 \mathrm{~mm}$ from the edge of the discontinuity. Axial direction (x-direction) is selected for extrapolating hot spot stress on the edge of the notch. The selection of the direction of hot spot extrapolation is made based on the assumption that the majority of stresses are acting in the axial direction.

The stress history obtained for the welded detail can later be processed using the rainflow counting algorithm, fatigue assessment or for any other post processing action.

The results of this numerical experiment show that the developed method can be used to determine the stresses of a structural detail using a real-time simulation model. This method enables a wide variety of uses from determining stresses from positions that cannot be measured from the real machine to determining the best practices for machine operation. Measuring bearing housing stresses, for example, in a real machine during an operation cycle is next to impossible. This method enables the determination of stresses during the entire operation cycle instead of just a suspected peak value. The method could be used to improve estimations on the machine durability as well as improving the machine durability already in the machine design phase. A practical example would be to use virtual prototyping in the machine product development phase. The model could then be used to run a series of reference operation cycles while recording displacement data from a structure. The recorded data could then be used to run analysis on several crucial parts of the structure in order to determine the life expectancy under operating conditions as well as different operators.

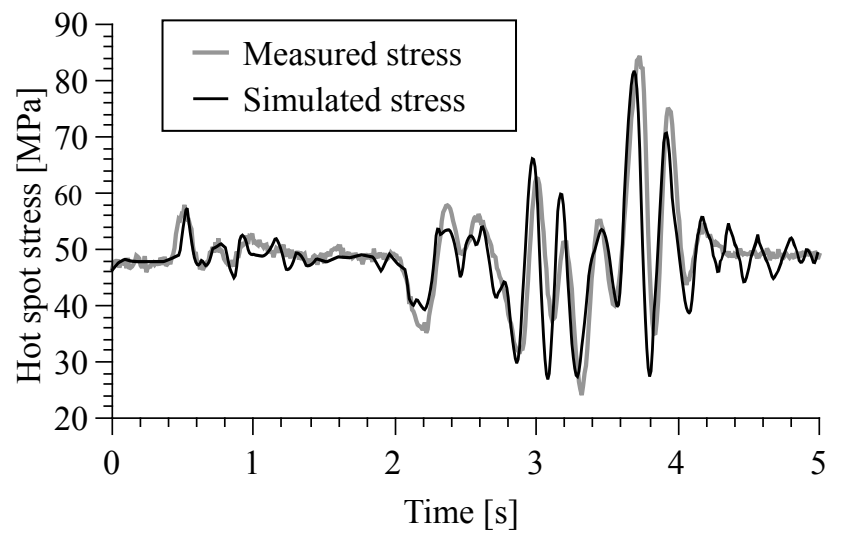

Figure 9. Stress history of a notch at the crane.

\section{Conclusions}

In this paper one approach of making fatigue analysis more usable among multibody simulation based product development is presented. In order to combine dynamic simulation and fatigue design this study introduces a novel approach for efficiently obtaining stress history from dynamic simulation.

This paper presents an approach in which the stress history for fatigue life estimation of an arbitrary structural discontinuity in a large-scale structure can be efficiently obtained in multibody simulation. In the proposed approach the structure is modeled with structured elements (i.e. planes or beams) in order to get rid small structural details to minimize nodal degrees of freedom. After that model is further reduced using component mode synthesis, in numerical example, CraigBampton method was used, this model is called as reduced model. Reduced model is used to represent flexible body in multibody simulation. Small structural details are modeled separately and are attached to reduced model using suitable methods. In this paper, in numerical examples sub-models were attached to reduced model using rigid beam webs. Submodels were analyzed quasi-statically within finite element codes using displacements, obtained from dynamic simulation, as boundary conditions. This analysis can be made during the dynamic simulation or in post-processing phase. Computations involving sub-modeling allow the fatigue assessment calculation to be separated from the dynamic simulation and structural details can be analyzed independently.

In future work the integration of fatigue analysis and produced stress history could be improved. The way how stress data is analyzed and compared to real fatigue test results differs from the stress results that can be straightforwardly obtained from dynamic simulation. In numerical examples, this aspect is taken into account using linear surface extrapolation to estimate hot-spot stress. The problem using that approach is the difficulty of knowing the most probable crack growth direction. In reality, crack may change the direction of growing depending on geometry and/or loading conditions. Also, 
in future work methods of attaching sub-model into simulation model should be studied carefully. Possibility to use coordinate reduction for sub-models and what kinds of limitations it provides to attachment for sub-models.

Acknowledgements. The research is supported by the Academy of Finland, project 133154.

Edited by: A. Müller

Reviewed by: J. Cuadrado and A. Schwab

\section{References}

Arczewski, K. and Frạczek, J.: Friction Models and Stress Recovery Methods in Vehicle Dynamics Modelling, Multibody Syst. Dyn., 14, 205-224, 2005.

Braccesi, C. and Cianetti, F.: A procedure for the virtual evaluation of the stress state of mechanical systems and components for the automotive industry: development and experimental validation, Proceedings of the Institution of Mechanical Engineers, Part D: Journal of Automobile Engineering, 219, 633-643, 2005.

Claus, H.: A Deformation Approach to Stress Distribution in Flexible Multibody Systems, Multibody Syst. Dyn., 6, 143-161, 2001.

Craig Jr., R. R. and Bampton, M. C. C.: Coupling of Substructures for Dynamic Analyses, AIAA J., 6, 1313-1319, 1968.

Dietz, S.: Vibration and fatigue analysis of vehicle systems using component modes, Doctoral thesis, Technische Universität Berlin, 1999.

Dietz, S., Netter, H., and Sachau, D.: Fatigue life predictions by coupling finite element and multibody systems calculations, in: Proceedings of Design Engineering Technical Conferences, 1997.

Dietz, S., Netter, H., and Sachau, D.: Fatigue Life Prediction of a Railway Bogie under Dynamic Loads through Simulation, Vehicle Syst. Dyn., 29, 385-402, 1998.

Haagensen, P. J. and Maddox, S. J.: IIW Recommendations on Post Weld Fatigue Life Improvement of Steel and Aluminium Structures, International Institute of Welding, Paris, 2011.

Jun, K., Park, T., Lee, S., Jung, S., and Yoon, J.: Prediction of fatigue life and estimation of its reliability on the parts of an air suspension system, Int. J. Automot. Techn., 9, 741-747, 2008.

Knight, N. F., Ransom, J. B., Griffin, O. H., and Thompson, D. M.: Global/local methods research using a common structural analysis framework, Finite Elem. Anal. Des., 9, 91-112, 1991.
Korkealaakso, P., Mikkola, A., and Rouvinen, A.: Multi-body simulation approach for fault diagnosis of a reel, Proceedings of the Institution of Mechanical Engineers, Part K: Journal of Multibody Dynamics, 220, 9-19, 2006.

Korkealaakso, P., Mikkola, A., Rantalainen, T., and Rouvinen, A.: Description of joint constraints in the floating frame of reference formulation, Proceedings of the Institution of Mechanical Engineers, Part K: Journal of Multi-body Dynamics, 223, 133-145, 2009.

Lee, S.-H., Park, T.-W., Park, J.-K., Yoon, J.-W., Jun, K.-J., and Jung, S.-P.: Fatigue life analysis of wheels on guideway vehicle using multibody dynamics, Int. J. Precis. Eng. Man., 10, 79-84, 2009.

Maddox, S. J.: Fatigue strength of welded structures, Abington Publishing, Cambridge, 2nd Edn., 1991.

Melzer, F.: Symbolic computations in flexible multibody systems, Nonlinear Dynam., 9, 147-163, 1996.

Miner, M. A.: Cumulative damage in fatigue, J. Appl. Mech., 12, 159-164, 1945.

Niemi, E., Fricke, W., and Maddox, S. J.: Fatigue analysis of welded components: Designer's guide to the structural hot-spot stress approach, Woodhead Publishing, Cambridge, 1st Edn., 2006.

Nikravesh, P. E. and Chung, I. S.: Application of Euler Parameters to the Dynamic Analysis of Three-Dimensional Constrained Mechanical Systems, J. Mech. Design, 104, 785-791, 1982.

Poutiainen, I., Tanskanen, P., and Marquis, G.: Finite element methods for structural hot spot stress determination - a comparison of procedures, I. J. Fatigue, 26, 1147-1157, 2004.

Shabana, A. A.: Dynamics of Multibody Systems, Cambridge University Press, Cambridge, 2nd Edn., 1998.

Shabana, A. A.: Dynamics of Multibody Systems, Cambridge University Press, Cambridge, 3rd Edn., 2005.

Tobias, C. and Eberhard, P.: Stress recovery with Krylov-subspaces in reduced elastic multibody systems, Multibody Syst. Dyn., 25, 377-393, 2011.

von Mises, R.: On Saint Venant's principle, B. Am. Math. Soc., 51, 555-562, 1945.

Wallrapp, O. and Wiedemann, S.: Comparison of Results in Flexible Multibody Dynamics Using Various Approaches, Nonlinear Dynam., 34, 189-206, 2003.

Watton, J.: Fluid power systems: Modeling, simulation, analog and microcomputer control, Prentice Hall, New York, 1st Edn., 1989.

Yim, H. J. and Lee, S. B.: An integrated CAE system for dynamic stress and fatigue life prediction of mechanical systems, J. Mech. Sci. Technol., 10, 158-168, 1996. 